Aus der dermatologischen Abteilung des Rudolf Virohow-

Krankenhauses in Berlin.

\title{
Über Impetigo herpetiformis gravidarum Hebrae und die Pathogenese der großen Schwangerschaftsdermatosen.
}

\author{
Von \\ San. Rat Dr. Wechselmann, \\ dirigierender Arzt.
}

(Hiezu die Kurven auf Taf. XII.)

Das Krankheitsbild der Impetigo herpetiformis in der Schwangerschaft und nach der Geburt ist von $\mathrm{Hebra}$ so scharf und klar gezeichnet worden, daß man, wie Scherber (1) mit Recht betont, bei der Beschreibung jedes neuen Falles darauf zurückgreifen soll. Die klassische Schilderung Hebras (2) lautet: „Die Erkrankung ist eine Proruption von mit Eiter gefüllten Bläschen, also Pusteln, welche sich sowohl durch ihren gleich bei deren Entstehen bemerkbaren eitrigen Inhalt, als durch ihre Anreihung, Gruppierung und periphere Ausbreitung besonders charakterisieren. Beinahe jedesmal zeigten sich die ersten Effloreszenzen an der Innenseite der Oberschenkel teils in kreuzergroßen Gruppen, teils vereinzelt stehend in Gestalt von stecknadelkopfgroßen Pusteln, die sich alsbald durch Nachschübe in der Peripherie zu Kreisen oder irisartigen Zeichnungen heranbildeten. Binnen wenigen Tagen vermehrte sich die Anzahl der Gruppen, erweiterten sich die Kreise und kamen immer neue vereinzelte Pusteln zum Vorschein, so daß nach und nach Oberschenkel, Bauch, Unterschenkel, Brust, Ober- und Vorderarme, Hände, Füße und zuletzt auch Nacken, Hals, Gesicht 
und behaarter Kopf mit solchen Pustelgruppen übersät erscheinen. Während die im Zentrum der einzelnen Gruppen gelegenen Pusteln zu flachen, dunkelbraunen Borken vertrockneten, entwickelten sich im Umkreise stets neue mit gelber eitriger Flüssigkeit gefüllte Effloreszenzen und ahmten somit das Bild eines Herpes iris oder circinatus nach." Die ganze Eruption und deren Verlauf waren von intensivem Fieber, trockener Zunge und großer Prostration begleitet. Bei drei Patienten dauerten die Nachschübe bis an das Lebensende ununterbrochen fort, während bei zwei anderen nach mehrwöchentlichem Bestande der Pustelgruppen und Kreise sämtliche Effloreszenzen vertrockneten und die endlich abgefallenen dicken Borken gesunde, allenfalls stärker pigmentierte Hautstellen hinterließen. Bei einigen wurde die epidermoidale Pusteldecke zu einem stinkenden Brei verwandelt; doch $\mathrm{kam}$ es auch hier nicht zu Ulzerationen. Von den 5 Fällen starben 4, einer heilte nach wiederholten Rezidiven; aber auch dieser ging nach Hebra junior suäter an einer Rezidive zu Grunde. Bei jedem Ausbruch der Pusteln waren Schüttelfröste zu beobachten, die dann einem mäßigen Fieber wichen, die Darmentleerungen vermehrt, der Urin enthielt kein Eiweiß. Von den 5 Patienten waren 2 im letzten Schwangerschaftsmonat, 3 waren $2-5$ Wochen nach der Entbindung. Daher glaubt H. die Grundursache der Erkrankung in einer krankhaften Veränderung des weiblichen Genitalapparats annehmen zu können. Die Sektion ergab kein puerperales Leiden; Erscheinungen von Syphilis waren in keinem Falle vorhanden.

Spätere Autoren, vor allen schon Kaposi (3), haben den Krankheitsbegriff erweitert, indem sie, im wesentlichen geleitet von der Morphologie des Exanthems, die Krankheit auch bei Männern oder auch bei Frauen unabhängig von den Vorgängen der Schwangerschaft und Geburt beschrieben. Mit Recht hat schon J a ris ch (4) dagegen Einspruch erhoben und S cherber uater Berücksichtigung der beschriebenen derartigen Fälle auch die klinische Verschiedenheit derselben betont. Dadurch schrumpft allerdings die Zahl der Fälle von klassischem $\mathrm{H} \mathrm{e} \mathrm{bra-}$ schen Typus auf ein Minimum ein; tatsächlich scheint aber auch die echte Impetigo herpetiformis eine sehr seltene Er- 
krankung zu sein, da außer Hebra und Kaposi alle anderen Autoren nur je einen Fall gesehen haben.

Dies rechtfertigt die ausführliche Mitteilung eines dem Hebraschen Typus genau entsprechenden Falles.

Die 21jährige Ehefrau P. wurde am 22. Mai 1907 auf der dermatologischen Abteilung des Rudolf $\mathrm{Vir}$ chow Krankenhauses aufgenommen. Sie war früher stets gesund, hat 3 normale Partus hinter sich; eines der Linder starb an Lebensschwäche, eines an Skrofulose, das dritte lebt. Die letzten Menses waren im September 1906 aufgetreten. Die Schwangerschaft verlief im Vergleich zu den früheren auffallend anders: es bestand starke Ermüdbarkeit und Schläfrigkeit, Ohrensausen, Frösteln und außerdem zeigte sich eine Veränderung der Psyche in Form eines auffällig reizbaren Wesens. Seit Weihuachten 1906 leidet die Patientin an einem Ausschlag, welcher zuerst an der Innenseite der Obexschenkel anftrat, sich dann auf der rechten, später auch linken Achselhöble und von da auf die Arme ausbreitete, später den Nabel ergriff und sich überall peripher ausbreitete. Vor etwa 14 Tagen wurden ihr vom Arzt Pillen und graue Salbe verordnet, wonach eine wesentliche Verschlimmerung des Ausschlags auftrat, welche sie veranlaßte, sich im Krankenhaus aufnehmen zu lassen. Pat. gibt an, daB sie in den letzten Monaten ofter gefiebert habe; die subjektiven Beschwerden, Jucken, Brennen, seien nicht sehr erheblich gewesen.

Stat. praes.: Mittelgroße, schwächlich gebaute, anämische Frau. Innere Organe ohne besonderen Befund. Urin klar, frei von Albumen und Sacharum. Augenhintergrund normal. Uterus etwas über handbreit über dem Nabel. Herztöne des Kindes deutlich hörbar; Rücken liegt rechts.

Die ganze Genitokruralregion, nahezu die ganze Innenfläche beider Oberschenkel, die ganze Bauchoberfläche, die Achselböhlen, die Innenseite der Oberarme bis zum Ellenbogen zeigt besonders in der Peripherie Gruppen, meist deutlich annulär angeordneter miliarer bis über hirsekorngroßer, flacher mit eiterähnlicher Flüssigkeit gefüllter Pustelchen; wăhrend das Zentrum der entzündeten Partien teils mit fetzig expolierter Epidermis, teils mit bräunlichen Borken belegt ist. Nach Abhebung dieser näßt der Grund kaum und ist nur mäßig gerötet; ja an manchen Stellen sieht man ohne Narbenbildung abgeheilte, wieder normale Haut. Sämtliche Herde zeigen den Charakter der Progression, indem die neuen Pusteleruptionen in der Peripherie sich bilden. Therapie. Feuchte Um. schläge, Boecksche Schüttelmixtur.

Am 24/V. stieg die bis dahin normale Temperatur unter Schüttelfrost auf 38' 6 unter Ausbruch neuer Pustelgruppen in der Peripherie der alten Herde. Permanentes Bad. In den nächsten Tagen bestand remittierendes Fieber. Am 27./V. wurde in Il. Schädellage ein unreifes Mädchen (Gewicht $2250 \mathrm{~g}$, Länge $42^{1} / 2$, Kopfumfang [groBer $31 \frac{1}{2}$, kleiner 29] entsprechend dem VII./VIII. Monat) geboren. Geburt und Plazentarausstoßung normal. Am folgenden Tage 28./V. starker Schüttelfrost, Temp. 
stieg auf 39.6, Puls 160. Aufschießen neuer Pustelgruppen am Rücken, Schulter und Klavikulargegend. Am 29./V. wurde durch Punktion der Vena cephalicasin. Blutentnommen und Blutagarplatten angelegt, welche sterilblieben; ebensowurden Kaninchen, Meerschweinchen undweibeMäusedamitsubkutanundintraperitoneal geimpft, ohne daBirgendeine Reaktion beiden Tieren eintrat. Auch derAustrich des Pustelinhalts zeigte keinerlei Mikroorganismen, nur zahlreiche polynukleäre Leukozyten und vereinzelte lymphozyten; Impfung von Agarrohrchen mit Pustelinhalt blieb gleichfalls ohne Effekt.

Am 30./V. zeigt sich am linken Unterarm gerade an der zum Zweck der Venaepunktion mit dem Gummischlauch abgeschnürten Stelle in der ganzen Circumferenz ein entzündlich geröteter Ring, in dessen Bereich zahlreiche mit trübem Inhalt gefüllte Bläschen sichtbar sind; der rechte Unterarm ist vollkommen unverändert, während sonst im Fortschreiten des Prozesses stets sehr auffallige Symmetrie herrschte.

2./VI. Anstieg der Temperatur auf 40.5 mit Schüttelfrost. Das Exanthem recidiviert auf früher schon ergriffen gewesenen und abgeheilten Stellen. Außerdem ist überall ein Fortschreiten des Exanthems nach der Peripherie sehr ausgesprochen.

10./VI. Schüttelfrost. T 40.1. Auftreten äußerst zahlreicher, dicht gedrängt stehender Pustelchen über dem ganzen Körper, auch im Gesicht, Fußsohlen und Hohlhand. Ödematöse Anschwellnng der Unterschenkel. Ausfallen der Scham- und Achselhaare. Nägel intakt.

Nochmalige Impfversuche des Pustelinhalts auf Nährböden ohne Erfolg. Urin frei von Albumen, Sacharum, Indikan. Diazoreaktion negativ Therapie. Mehrstündiges Wasserbad und Thiopinolzusatz. Borvaseline. Tr. Strophanti.

14./VI. Starker Durchfall. Starkes Brennen im Rachen und in der Speiseröhre. Ausschlag am Körper fast geschwunden. Tr. opii.

15./VI. Neuer Temperaturanstieg mit Ausbruch über Stamm und Extremitäten. Deutlicher Bläschenausbruch an der Unterfläche der Zunge.

Nochmalige Venaepunktion im Frostanfall and An. legung von Blutagarplatten, welche steril bleiben.

Nach einer wesentlichen Besserung, Abfall des Fiebers, Rückkehr des Pulses zur Norm während etwa einer Woche, Rückfall am 20./VI. 21./VI. Pustelinhalt steril. 26./VI. 40.5. Neue Eruptionen an Hals, Brust, Abdomen, aber auch Zunge und Lippenschleimhaut. Dabei Brechreiz und Erbrechen.

29./VI. Abfall der Temperatur steil auf $37^{\circ}$. Besserung des Allgemeinbefindes, dem ảber wieder vom 1.-4./VII. ein Rückfall folgt. Nach einem Intervall bis zum 7./VII. bei sehr gutem Allgemeinbefinden traten wieder explosionsartig am ganzen Körper (auch an der Zunge) Eruptionen auf unter hohem Fieber bis zum 14./VII. Danach nur noch Rötung der 
Haut, keine Pusteln und fieberfreier Intervall bis zum 17./VIT., dem ein heftiges Rezidiv mit Bläschen an den Beinen, Zunge und hartem Gaumen, zeitweiser Somnolenz, hohem Fieber, mit eintägiger Unterbrechung am 24./VII., bis zum 28./VII. folgte. Am 27./VII. Infusion von $300 \mathrm{cbm} \mathrm{NaCl-}$ Lösung. Am 28./VII. steiler Abfall. 29./VII. fieberfrei, dann wieder hohes Fieber. Dabei gutes Allgemeinbefinden. Am 1./VIII. Never Ausbruch auch auf der jetzt zum Teil haarlos gewordenen Kopthaut. Kein Albumen, keine Diazoreaction. Am 5./VIII. kritischer Fieberabfall. Von da an ganz normale Temperaturen und guter Puls bis zum 10./VIII, wo Pat. auf Wunsch entlassen wurde. Es bestand nur noch Rötung an der Haut der Streckseiten der oberen und unteren Extremitäten, sonst war die Haut normal. Die mehrfach von uns und Herrn Dr. Hans Hirschfeld vorgenommene Blutuntersuchung ergab normales Verhalten, spez. keine Eosinophilie. Nach unseren Erkundigungen trat Patientin in Behandlung des Herrn Kollegen O. Rosenthal, wo sie unter indifferenter Therapie bis Oktober völlig heilte und fernerhin auch gesund blieb.

Am 29./VI. 09 suchte sie wiederum die Poliklinik des Herrn Kollegen Rosenthal, dessen Liebenswürdigkeit ich die folgenden Daten verdanke, auf. Sie war im IX./X. Monat gravida und litt seit 3 Wochen an einem Ausschlag. Effloreszenzen an den Streckseiten beider oberen Extremitäten von der Hälfte der Oberarme bis zur Mitte der Unterarme. Am Abdomen vereinzeite größere und kleinere Effloreszenzen; Oberschenkel wenig, Unterschenkel reichlich befallen und zwar die Beugeseite. Die Effloreszenzen sind herpesartige Eruptionen und Pusteln. Diagnose: Impetigo herpetiformis.

Am 16./VH. Partus. Danach Verschlimmerung besonders am rechten Unterschenkel. Da diese anhält, Aufnahme am 27./VL. in die Klinik. Status vom 28./VII. Hochgradige Magerkeit, mäßige Blässe. Urin frei von Eiweiß und Zucker. Umschriebene Stellen von Dermatitis exfoliativa, zahlreiche hirse- bis reiskorngroße Literpusteln vorwiegend in der Umgebung der Exkoriationen, aber auch an sonst intakten Hautstellen. Der Krankheitsprozeß hat besonders stark die Extremitäten ergriffen. R. Bein zeigt an der Außenseite von der Mitte des Oberschenkels bis handbreit unter den Condylus externus eine streifenförmige, handbreite, exfolierte Fläche; eine gleiche handfächengröbe an der Innenseite des Oberschenkels; am Unterschenkel einige 2-3 markstückgroße Herde. Linkes Bein: zwei etwa 2markstückgroße und mehrere kleinere Herde am Oberschenkel, eine größere Anzahl kleinerer am Unterschenkel. An den oberen Extremitäten rechts, Ober- und Unterarm von verschieden großen Herden befallen, am Ellenbogen etwa handgroße Exfoliation; desgl, am linken Ellenbogen. An der 1. Hüfte, Bauch, Brust und Rücken nur vereinzelte kleine Herde. Kopfhaut intakt; kein Defluvium.

Besonders in der Umgebung der erkrankten Stellen traten frische Impetigobläschen auf, welche wieder zur Exfoliation führten. Aber auch auf vorher ganz intakten Hautstellen z. B. auf der Brust, dem Hals, an der Stirnhaargrenze, an Händen und FüBen traten Eruptionen auf, so daß 
nach und nach der ganze Körper (auch Lippen, Zunge, Mundschleimhaut und Kopfhaut) das Bild der Dermatitis exfoliativa darbot.

Das Allgemeinbefinden war bei der Aufnahme sehr schlecht; andauernde Temperaturen zwischen $38-39^{\circ}$; öfteres Erbrechen, häufig Verweigerung der Nahrungsaufnahme. In der 2. Woche trat nach einem schweren Diätfehler erhebliche Verschlechterung auf. Temperatur schwankte 9 Tage hindurch zwischen 39 und $40^{\circ}$; Pat. erbrach fast jede Nahrung und batte bäufige Durchfälle. Nachdem der ProzeB auf der Haut zum Stillstand gekommen war, ging die Temp. anf $38^{\circ}$ zurück, der Puls besserte sich, die Durchfälle hörten auf, Temp. wurde normal, Appetit stellte sich ein und Pat. fühlte sich subjektiv wohl. Dagegen zeigte sich jetzt im Urin 5\% Albumen. Fine Woche vor der Entlassung stieg der Eiweißgehalt auf $1 \%$, während kleine Rezidive auf der Haut der Ellenbogen und Fübe auftraten, sowie Erbrechen, Durohfall. Das Allgemeinbefinden verschlechterte sich, es traten Ödeme der Unterschenkel auf; Pat. ließ Urin unter sich. Temp. 37.5-36.5. In diesem Terminalstadium wurde sie am 30./VIII. im Rudolf Virchow-Krankenhaus aufgenommen, wo sie unter Fieber $37-39^{\circ}$ und Erscheinungen von Somnolenz am 3. IX. starb.

A us dem Sektionsbericht sei erwähnt: Im Herzbeutel zirka $50 \mathrm{ccm}$ klare Flüssigkeit. Zwischen der Vorderwand des Herzens und dem Herzbeutel geringe Verwachsungen.

Herz anämisch, Klappen intakt.

Inhalt des Herzens: Speckgerinsel.

Beide Lungen sind der Brustwand adhärent. Lungengewebe im Unterlappen leicht hyperämisch und ödematös. Milz zicmlich groß, derb, Follikel deutlich sichtbar, etwas glasig.

Magen und Pankreas o. B.

Muskatnußleber, trübe Schwellung derselben.

Uterus etwas vergrößert, ziemlich weich. Schleimhaut etwas geschwollen, aufgelockert, zum Teil mit schmierig gelben Belegen bedeckt. Rektumschleimhaut injiziert geschwollen. Follikelzeichnnng sehr deutlich! Linke Niere etwas vergrößert, Kapsel schwer abziehbar. Oberfläche glatt. Rinde auf dem Durchschnitt verbreitert, trüb überquellend. Vereinzelte Blutungen und Nekrosen. Markzeichnung dentlich scharf gegen die Rinde abgesetzt. Nierenbecken frei.

Rechte Niere etwas weniger blutreich als die linke, zeigt die gleichen Degenerationsprozesse etwas stärker ausgesprochen.

Nebennieren ohne Befund.

Im Dickdarm und den unteren Dünndarmabschnitten Rötung und Schwellung der Schleimhaut.

Halsorgane, große Gefäße ohne Befund.

Der beschriebene Fall entspricht vollkommen dem Hebra schen Typus. Die Krankheit beginnt bei der 21 jährigen Frau im 4. Monat der 4. Schwangerschaft, steigert sich sehr nach der 
Entbindung und heilt endlich nach vielen Nachschüben nach etwa 10 Monaten aus, um in der nächsten Schwangerschaft nach 2 Jahren prompt wieder $z u$ erscheinen und nach der Entbindung unter vielen Nachschüben zur allgemeinen Erythrodermia exfoliativa und zum Tode zu führen. Die Geburten selbst boten nichts Besonderes, ebenso wenig die Kinder. Der Ausschlag begann typisch an der Innenseite der Oberschenkel und entsprach in Aussehen und Verlauf der Schilderung Hebras. Sehr ausgesprochen war vielfach das symmetrische Fortschreiten besonders an den Extremitäten; es scheint jedoch, daß die Schädigung der Haut schon angelegt ist, ehe es zum Auftreten des Exanthems kommt, da der Druck der zur Venaepunktion angelegten Gummibinde genügte, um am Vorderarm das Exanthem hervorzurufen, während es an der symmetrischen Stelle des anderen Armes erst einige Tage später nachfolgte. Erwähnenswert ist der Ausfall der Kopf-, Scham- und Achselhaare, welcher auch in dem Fall Glaereckes erwähnt ist. Der Ausgang in herdweise und schließlich allgemeine Erythrodermie hat seine Analogie in den psoriasiformen Exanthemen Dubreuilhs.

Das Befallenwerden der Schleimhäute ist in vielen Beobachtungen erwähnt.

Erwähnenswert sind die starken zerebralen Symptome, die schon vor Fintritt des Exanthems als besondere Schläfrigkeit und auffallende Reizbarkeit auftraten, dann aber auch während der ganzen Krankheit anhielten. Man könnte wegen des häufigen Erbrechens an Tumoren des Gehirns speziell der Hypophysis denken; der Augenhintergrund war aber normal; Sektion des Schädels war nicht möglich.

Der Urin war bis in die letzte Zeit normal, erst dann kam es zur Albuminurie. Der Blutbefund war beim ersten Krankenhausaufenthalt stets normal.

$\mathrm{Zu}$ der bekannten besonders ron du Mesnil, Borzecki, Krzystallowitz, Scherber geschilderten Histologie der Hauteffloreszenzen können wir nichts beitragen, weil Patientin eine Exzision verweigerte. In der letzten Lebenszeit bestand das Bild einer allgemeinen exfoliativen Erythrodermie; dem- 
entsprechend zeigt das histologische Bild eine starke, z. Th. fingerförmige Wucherung der Papillen. Das Stratum corneum ist lamellös zerklüftet und fast überall rom Stratum lucidum fetzig abgelöst. Das Stratum granulosum besteht zumeist aus einer Zellage. Die Grenzen der anderen Retezellen sind undeutlich, die. Interspinalräume nicht wesentlich verbreitert, die Kerne blaß z. Th. ron Vakuolen umgrenzt. Überall Pigment unregelmäßig zerstreut.

Das Bindegewebe färbt sich mit allen Farbstoffen auffallend schlecht, so dab es als etwas entartet (hyalin?) angesprochen werden darf. Die elastischen Fasern zeigen kaum Veränderungen. Die Papillargefäße und die oberflächlichen des subpapillaren Netzes sind erweitert und mit einem aus Lympozyten und Bindegewebszellen gebildeten Mantel umscheidet. Sehr viele dieser länglichen Bindegewebszellen, besonders auch in der Umgebung der normalen Follikel und Schweißdrüsen sind bei Färbung mit polychromem Methylenblau leuchtend rot. Doch lassen sie die Körnelung der Mastzellen vermissen und stellen sich mehr als schollige Bildungen mit einem runden, Farbstoff nicht annehmenden, glänzenden, an degenerierte Kerne erinnernden Einschluß dar.

Färbungen auf Bakterien ergeben ein negatives Resultat. Leukozyten nur sehr spärlich. Keine Plasmazellen.

Die Gefäße des Uterus zeigen eine sebr starke hyaline Degeneration der Gefäße, spez. der Muskularis.

Die Leber zeigt ihre Zellen mit großen glänzenden Fetttropfen erfüllt: starke Fettinfiltration.

Die Nieren zeigen die Epithelien der Harnkanälchen unregelmäßig gequollen, getrübt, zum Teil losgelöst, auch sind vielfach hyaline Zylinder zu sehen. Das Bindegewebe ist verbreitert mit Einlagerung von einkernigen Rundzellen. Die Glomeruli zeigen in ihren Epithelien die gleichen Veränderungen u. sind meist mit amorphen, an die Schlingen erinnernden Massen, in welchen Kerne vereinzelt und unregelmäßig zerstreut liegen, erfüllt, welche alle Amyloidreaktionen ergeben. Desgleichen zeigt die Milz neben ungefärbten Herden mit verwischter Struktur ausgedehntes Amyloid. 
Wie bereits eingangs hervorgehoben wurde, weisen die beim Manne oder außer Zusammenhang mit der Schwangerschaft beschriebenen Fälle von I. h. derartige Differenzen gegen den klassischen Hebraschen Typus auf, daß es bis auf weiteres berechtigt erscheint, zur sicheren Diagnose der echten I. h. Hebrae neben den klinisch charakteristischen Symptomen Zusammenhang der Krankheit mit den Vorgängen der Schwangerschaft und der puerperalen Involution zu fordern. Es erscheint sogar bei dem derzeitigen Stand unserer Kenntnisse, um in das Wesen der Krankheit einzudringen, vorteilhafter, die Morphologie des Exanthems nicht zu sehr in den Vordergrund zu rïcken, sondern vielmehr die an Schwangerschaft und Wochenbett geknüpften großen Dermatosen: allgemeine Erytheme, Herpes gestationis, Pemphigus gravidarum, Imp. herpetiformis als ätiologisch einheitliche Krankheiten aufzufassen. Dafür spricht, daß diese "Schwangerschaftsdermatosen" zumal Pemphigus, Imp. herp. und polymorphe als Dermatitis herp. Duhringi bezeichnete, zugleich oder abwechselnd mit einander auftreten können, so daß die Wahrscheinlichkeit nahe liegt, daß dieselbe Noxe verschiedene Exantheme erzeugt und die Formen derselben nur graduelle Unterschiede darstellen; die Verschiedenheit der Prognose, speziell der fatale Ausgang, welcher bei der I. h. die Regel zu sein scheint, bildet keinen prinzipiellen Unterschied, da ja der Tod meist erst in späteren Rezidivanfällen eintritt, aber auch andere grobe Schwangerschaftsdermatosen ab und zu tödlich verlaufen. Ein vorzügliches Beispiel für das gleichzeitige Vorkommen mehrerer Schwangerschaftsdermatosen bildet ein Fall von Pospelow, welcher nur in den Berichten der Moskauer dermatologischen Gesellschaft 1897/98 russisch veröffentlicht und daher nicht genügend bekannt wurde; ich gebe ihn daher seines auch in anderer Hinsicht großen Interesses wegen ausführlich wieder.

Die 32jührige Patientin wurde am 12. Nov. 1897 in die Klinik aufgenommen; bis zu ihrer Verheiratung im 20. Lebensjahre zeichnete sie sich durch eine gute Gesundheit aus. Sie war sechsmal schwanger; die erste Schwangerschaft wurde durch einen Sturz aus dem Wagen unterbrochen, doch das Kind lebend geboren; es starb nach 11 Monaten aus einer unbekannten Ursache. Die 2. und 3. Schwangerschaft endeten zur rechten Zeit, aber die Kinder starben nach $5-6$ Tagen. Die 4. Schwan- 
gerschaft endete mit Abort im 2. Monat. Dann wurde ein Knabe geboren, der z. Z. $4^{1} \frac{1}{2}$ Jahre alt war. In der 6 . Schwangerschaft vor $2^{1 / 2}$ Jahren kam es im 2. Monat zur AusstoBung einer Blasenmole. Nach 3 Wochen ein hartnäckiger Blutverlust, der sich infolge von heißen Spülungen verringerte und nach Auskratzung der Gebärmutter aufhörte. Kaum aber hatte die Kranke das Bett verlassen, so fing nach 3 Tagen das Blut wieder an sehr heftig zu fließen und dies hörte nicht mehr auf. Im Mai 1897 wurde der Blutverlust wieder stärker und erst im Oktober geringer; die Ärzte nahmen ein Uterusfibroid an. Die Blutungen schwächten die Patientin enorm; sie verlor Schlaf, Appetit und wurde in hohem Maße nervös, erregbar und erschöpft, so daß sie gänzlich bettlägerig die Klinik aufsuchen mußte. Ende Oktober 1897 zeigte sich um den Nabel herum, nachdem Brennen und Schmerz in der Haut vorausgegangen war, ein Ausschlag von "Bläschen", welcher in der Mitte $\mathrm{zu}$ einem Schorf eintrocknete, in dessen Umgebung sich dieselben "Bläschenhäufchen" bildeten. Nach einer Woche verbreitete sich der Ausschlag über den ganzen Bauch, Hals, Räcken, obere und untere Extremitäten. Auch hier bildeten an einzelnen Stellen die Bläschenhäufchen einen Schorf, aber ein Teil von ibnen, der sich mit einem serösen fast durchsichtigen Inhalt erfüllte, bildete Blasen, welche die Größe einer Walnuß und darüber erreichten. Dabei bestand sehr heftiges Jucken, so daß Patientin einen Teil der Blasen aufkratzte.

Bei der Aufnahme in die Klinik am 12. Nov, 1897 bot die Kranke an den genannten Stellen das typische Bild der Impet. herp. Hebra dar; es werden Gruppen von Bläschen mit träbem Inhalt, welcher sehr schnell eitrig wird, beobachtet; sie heilen im Zentrum mit Bildung eines Schorfes $a b$, in dessen Perjpherie auf geröteter Haut neue Bläschengruppen, z. T. in annulärer Anordnung aufsprießen. An den unteren und besonders oberen Extremitäten wechseln Gruppen solcher Pustelchen mit walnußund darüber großen, mit durchsichtiger oder opaleszierender Flüssigkeit gefüllten Blasen ab. Herpetische Ausschläge z. T. in Gruppen z. T. einzeln befinden sich auch auf den Lippen, der Zunge, dem weichen Gaumen und nach den Schmerzen, die sie beim Schlucken der Speisen hat, auch auf der Schleimhaut der Speiseröhre. Von den übrigen Organen ist zu erwähnen, daß der Uterus um das vierfache vergrößert, derb ist und noch mäßig blutet. Lungen ohne Veränderungen. Atmung beschleunigt. Puls 130. Temp. morgens 37.9, abends 38.2. Trockene Zunge, großer Durst, Kopfschmerz, große nervöse Unruhe, Schlaf losigkeit, starkes Jucken an Händen und Beinen, starke Erschöpfung. In den nächsten Tagen stieg unter Schättelfrost die Temperatur von 37.4 auf 40.1 und fiel am 5. Dez. wieder anf 37.5. Dabei Ausbruch neuer Pustelchen. Neue Ausbrüche traten danach nur in geringem $\mathrm{Maßje}$ an den unteren Extremitäten auf, verschorften und überhäuteten. Am 21. Nov, traten Schmerzen in der Brust, blutiger Auswurf auf; Perkussion negativ, Auskultation Giemen nnd Rhonchi; keine Tuberkelbazillen im Auswurf. Am 1. Dez. heftige Kopfschmerzen, Erbrechen, Puls 130, Schlaflosigkeit, Schwäche, Bewußtlosigkeit, die bis 
zum Tod am 7. Dez. anhielt. Bei der Sektion zeigte sich ein Deziduoma, welches die Gebärmutter beträchtlich vergrößert und Metastasen in Lungen, Leber, Milz, Nieren, Darm und im Hirn- und Rückenmark gesetzt hatte; auch in der Haut wurden hin und wieder kleine Deziduomknötchen gefunden.

Müssen wir also für die echte I. h. einen direkten $\mathrm{Zu}-$ sammenhang mit den Vorgängen der Schwangerschaft, mag dieselbe auch als Blasenmole ohne Foetus verlaufen, fordern, so hat $S c h e r b e r$ den Begriff der I. h. noch mehr eingeengt, indem er als Kriterium forderte: Sterilität des Pustelinhaltes und Blutes.

Wir glauben nach unseren jetzigen Kenntnissen durchaus $S \mathrm{ch}$ e r b e r zustimmen zu müssen, wenn er sagt: „In allen Fällen, wo sich Eitererreger fanden, handelte es sich entweder direkt um einen Staphylokokken- oder Streptokokkenprozeß, von welchen Infelitionen ohne weiteres zugegeben werden soll, daß sie der Imp. herp. klinisch ähnliche, aber doch stets deutliche Differenzen aufweisende Bilder zu erzeugen im Stande sind."

Allerdings wird dann die Zahl der sicheren, weil genau in dieser Hinsicht untersuchten Fälle von $I$. h. eine minimale und beschränkt sich auf die Beobachtungen von Freyhan, Breier, Maret-Gunsett, Nobl, Scherber, da auch der nach Scherbers Arbeit publizierte Fall Gavazzenis (5), der auch eine Virgo betraf, im Pustelinhalt wiederholt Streptococcus pyogenes zeigte. In unserem Falle ist die Sterilität des Pustelinhalts ebenso wie die des zumal auch während des Schüttelfrostes entnommenen Blutes durch Färbung, mehrfache Aussaaten und Tierimpfungen einwandsfrei erwiesen.

Danach muß man wohl als ätiologisches Moment eine Infektion fallen lassen. Hebra selbst stand auch auf diesem Standpunkt und die gelegentlich beobachteten puerperalen Infektionen können schon deswegen ätiologisch keine Rolle spielen, weil ja die I. h. schon in der Schwangerschaft beginnt. Als andere ätiologische Möglichkeiten sind dann noch hypothetisch neurogene und toxische Ursachen für die Pathogenese der I. h. ganz allgemein herangezogen worden. Hebra selbst stand noch so sehr unter dem gewaltigen Eindruck der Cla u deBernardschen Lehre, daß er in dem Rezidivieren des Pem- 
phigus in der Schwangerschaft ein Zeichen von Hysterie sah; heut, wo die erfahrensten Gynäkologen, z. B. Zweifel und Olshausen, selbst für die Hyperemesis gravidarum jeden Zusammenhang mit Hysterie ablehnen, wird man bei einem so schweren Symptomenkomplex, wie ihn die I. h. darstellt, in dessen Vordergrund Schüttelfröste, hohes, monatelang anhaltendes Fieber und fast stets tödlicher Ausgang stehen, nicht im entferntesten mehr an neurogene Einflïsse denken können. Bleibt demnach schon nach theoretischen Erwägungen - wenn man nicht gänzlich unbekannte und mit unseren Methoden nicht darstellbare Bakterien zur Erk]ärung heranziehen will allein die Intoxikationshypothese für den Ursprung der $I . ~ h$. übrig, so sprechen auch alle Symptome deutlich dafür. Dementsprechend haben auch einzelne Autoren (Vidal) (6) die Schwangerschaftsdermatosen als Autointoxikationen aufgefaßt; abgesehen aber von der scharfen Kritik, welche der ganze Begriff der Autointoxikation neuerdings erfahren hat (A. Schmidt), müßte stets noch eine besondere, nicht näher erklärte Empfindlichkeit gerade des schwangeren Organismus für die ja auch sonst auftretenden Gifte als Mittelglied eingeführt werden. Nun ist aber der Zusammenhang der I. h. (Fälle von D u Mesnil, Schulze, Nobl, Canuet etc.) (7) und der anderen großen Schwangerschaftsdermatosen mit Schwangerschaft und Wochenbett ein so klar ausgesprochener, daß er schon ganz alten. Beobachtern auffiel (Literatur bei Perrin) (8); als typisches Paradigma möge folgende drastische Schilderung $\mathrm{Hebras}$ angeführt werden.

Hebra beobachtete eine Russin, welche während dreier Graviditäten an Pemphigus gelitten hatte. Das Exanthem war im 5. Monat der ersten Schwangerschaft zuerst aufgetreten urd schwand nach der Geburt; in der 2. Schwangerschaft trat es im 3 . Monat auf und dauerte im Wochenbett einen Monat lang. Bei der dritten Schwangerschaft, welche mit der Geburt eines toten Kindes endigte, wurde der Pemphigus durch stets neue Nachschübe chronisch, heilte aber unter Hebras Behandlung in Wien. Die Angst, wieder schwanger zu werden, wenn sie sich nach Hause begeben würde, bestimmte die Patientin, den nächsten Winter in Wien zu bleiben, wo die Heilung anhielt. Ihr Gemahl, der große Sehnsucht nach seiner Frau trug, bestürmte sie wiederholt, in seine Arme zu eilen; aber sie blieb standhaft fern von der Heimat. Im nächsten Frühling aber überraschte der Gatte plötzlich seine geliebte Frau und die unausbleib- 
liche Folge der Vereinigung beider Eheleute ließ nicht lange auf sich warten. Schon im 1. Monat, bevor noch durch Ausbleiben der Menses der Verdacht auf stattgehabte Konzeption rege wurde, zeigten sich einzelne Blasen auf den unteren Extremitäten, welche nach Mabgabe der vorrückenden Schwangerschaft sich auch auf die obere Extremität verbreiteten; Appetit und Schlaf lagen darnieder, die Patientin magerte ab und verfiel in den alten Zustand. Nach der Geburt eines gesunden Knaben verlor sich der Pemphigus. Später gebar sie noch zweimal und wurde jedesmal während der Gravidität von Pemphigus heimgesucht, welcher bis einige Zeit über das Wochenbett hinaus dauerte.

Bei diesem so deutlich ausgesprochenen Zusammenhang der oben angeführten Dermatosen mit der. Schwangerschaft und dem Wochenbett liegt es gewiß nahe, ätiologisch an bestimmte Schwangerschaftsgifte zu denken. Dafür spricht vor allem der Umstand, daß eine für die Mütter so verderbliche Krankbeit, wie die I. h., die Kinder nicht wesentlich schädigt. Die Forschungen der letzten Jahre haben festgestellt, daß Auswurfstoffe des Foetus, wie auch Teile der Plazenta in die Zirkulation der Mutter gelangen. Vor allem werden Chorionzotten nicht nur bei Eklampsie [Schmorl (9), Veit (10)], sundern auch sonst vielfach bei Schwangeren deportiert. Nach den Forschungen von Veit und Scholten (11), Weichard (12), Liepmann (13) u. a. ist anzunehmen, daß diese in die Zirkulation der Mutter gelangten Synzytialzellen durch Zytolysine aufgelöst werden und dabei auch stark giftige Substanzen, Synzytiotoxine, entstehen. Die Plazenta, welche das Assimilationsorgan der mütterlichen Nährstoffe für den Foetus darstellt (Hofbauer) (14), ist ausgezeichnet durch ihren hohen Gehalt an Fermenten der verschiedensten Art, welche durch Deportation von Chorionzotten, aber wohl auch gelöst, in den mütterlichen Organismus gelangen. Gräfenberg (15) hat nachgewiesen, daß die Chorionzotten der ersten 3 Schwangerschaftsmonate ein tryptisches Enzym enthalten, welches im 4. Monat abnimmt und schwindet - gleichzeitig mit der Beendigung der Eieinbettung; er bringt daher die Vorgänge bei der Eieinbettung, welche nach den neueren Anschauungen als ein Einfressen der Eieinlage in das mütterliche Gewebe aufgefaßt wird, mit diesem stark wirkenden tryptischen Ferment in Beziehung; dafür spricht auch, daß mit Beendigung der Plazentation das 
eiweißlösende Enzym aus den Chorionzotten verschwindet, weil es seine Funktion erfüllt hat.

Durch die Untersuchungen von Walter Loeb und Shigeji Higuchi (16) ist uns aber auch ein genauerer Einblick in die Fülle der in der Plazenta vorhandenen Fermente eröffnet worden. Die blutfreie Plazenta enthält danach im frischen und im trockenen Zustand Katalase und Oxydase, sowie Stärke und Glykogen, spaltende Diastase. Inulase und Invertase sind hingegen nur im frischen Plazentarbrei wirksam, nicht im trockenen Pulver. Laktase, lipolytische und glykolytische Enzyme sind im frischen Brei und im trockenen Pulver nicht vorhanden. Enzyme von der Wirkungsart des Pepsins sind im Brei und im Pulver, solche, die Eiweiß bis zum Tyrosin abbauen, nur im ersteren enthalten, während Enzyme vom Typus des Erepsins, der Urease und Desamidase fehlen. Besonders wichtig ist dann noch das von $\mathrm{Higu} \mathrm{chi}$ besonders bearbeitete Fibrinferment (17). Man kann als erwiesen annehmen, daß ein Teil dieser Fermente, sicher wohl das tryptische, in den mütterlichen Organismus gelangt; denn nach den Untersuchungen Gräfenbergs ist der Antitrypsingehalt des Blutes jeder Schwangeren etwa doppelt so groß als der Nichtschwangerer, er schwankt zwischen 6-9:1 gegenüber dem Normalwert $4: 1$. Diese Angaben sind von Becker (18) für ca. $50 \%$ der Schwangeren bestätigt und besonders fand er bei 30 Frischentbundenen nur 4mal normalen Antitrypsingehalt, 5mal Vermehrung und $21 \mathrm{mal}$ starke Steigerung der trypsinhemmenden Kraft, womit Beobachtungen von Jochmann (19) und Gräfenberg übereinstimmen. Jochmann hat num nachgewiesen, daß die Einverleibung von Leukozytenferment sowohl wie von Pankreastrypsin bei Tieren nach vorübergehender Antifermentrerminderung, die durch Absättigung eines Teils des Antitrypsins bedingt ist, alsbald eine Steigerung der Antifermentmenge verursacht, und da 3 man die Steigerung durch öftere Wiederholung und steigende Dosen von Ferment nach Art eines Immunisierungsvorgangs noch beträchtlich erhöhen kann.

Danach ist der vermehrte Antitrypsingehalt nun stets bedingt durch eine Reaktion des Körpers auf einen irgendwo im Körper vorhandenen Trypsinreiz, daher im wesentlichen 
abhängig vom Leukozytenferment, rom Pankreastrypsin, bei Schwangeren rom Plazentaferment und bei den Frischentbundenen von der Anhäufung von Leukozytenferment an der Plazentarstelle und im Kolostrum, nach Beckers Vorstellung auch durch Zerfall verschleppter Chorionzotten.

Nun ist es aber in hohem Maße bemerkenswert, dal bei der Impetigo herpetiformis, aber auch bei den anderen Schwangerschaftsdermatosen, z. B. Herpes gestationis, die bestehenden Erscheinungen nach der Entbindung fortdauern, eine jähe Steigerung erfahren oder aber selbst die bereits geschwundene Krankheit mit großer Heftigkeit wiederkehrt.

Dieser Umstand tritt schon in Hebras Beobachtungen von Impetigo herpetiformis, besonders in dem von $\mathrm{Geber}$ (20) ausführlich veröffentlichten Fall klar hervor. Ebenso in vielen der von Dubreuilh (21) veröffentlichten Fällen Kaposis, besonders in Fall 2.

29jährige Fran, 10. Gravidität. Im 8. Monat erster Ausbruch der Impetigo herpetiformis. Entbindung am 16. Tage nach Beginn der Erkrankung. Am 18. rapide Ausbreitung des Exanthems, am 19. Parese der rechten oberen Extremität, am 20. tonische und klonische Krämpfe, am 23. Delirien und Krämpfe. Später Nackensteifigkeit, Koma, anhaltendes Erbrechen von grünen, später blutigen Massen und Tod.

Im Falle von Freyhan trat die Krankheit erst am 8. Tage des Wochenbetts auf, im Falle Sabolotzkys erkrankte die Frau 2 Tage vor der Entbinaung und ihr Zustand verschlimmerte sich am 8 Wochenbettstage; die meisten Fälle, so z. B. auch der Fall Scherbers und der unsrige, nehmen ihre tödliche Wendung erst im Wochenbett. Danach muß auch der Vorschlag Glaeveckes, bei I. h. die künstliche Frühgeburt einzuleiten, sehr zweischneidig erscheinen.

Am deutlichsten ausgesprochen ist dieses Aufflammen im Wochenbett beim Herpes gestationis, bei welchem dies nach Brocq am 4. bis 5. Wochenbettstag fast typisch ist, aber auch bei Pemphigus and Dermatitis herpetiformis Duhring, wofür auch folgende Beispiele angefübrt seien.

Bunol (bei Brocq) (22): Herpes gestationis, Beginn im 5. Monat der 2. Gravidität, welcher 12 Tage dauert, in den folgenden Monaten der Schwangerschaft nur eine geringe Menge von Vesikeln bedingt. Vier Tage nach der Entbindung trat ein neuer Ausschlag auf. Wiederholung 
des Exanthems im 5. Monat der 3. Gravidität und am 4. Tage nach der Entbindung dasselbe bei der 4. Gravidität.

Köbner (23): Ausbruch eines Pemphigus unter Fieber am 2. bis 3. Tage nach der ersten Entbindung. Kurz vor Ablauf der zweiten Schwangerschaft verspürte sie heftiges Brennen. Am Tage nach der Entbindung Schüttelfrost, Hitzegefühl, unerträgliches Jucken, Hautröte und an den folgenden Tagen schubweise Entwicklung von Vesikeln und Blasen.

Wyndham Cottle zit. bei Brocq. Za Beginn des 4. Schwangerschaftsmonats pruriginöser Ausschlag von Knötchen, Vesikeln und Blasen; sehr heftiger Anfall am 4. Tag nach der Entbindung.

Stanley Gale zitiert bei Brocq. Aussehlag erschien gewöhnlich 4 Tage vor der Entbindung und verschlimmerte sich allmäblich bis in die 3. Woche nach der Geburt. Ähnlich in dem von Brocq zitierten Fall von Walter G. Smith, Oswald, Sabin, 2mal am 3. Wochenbettstage, Radcliffe Crocker (3. Tag).

Noch eklatanter sind Lessers Fälle (Buschke) (24). Frau in der 6. Gravidität erkrankt an Herpes gestationis. Ausheilung, aber 14 Tage nach der Niederkunft neuer Ausbruch und Nachschübe durch $3 / 4$ Jahre: Gesundheit durch zwei Jahre; neuer Ausbruch bei neuer Gravidität, der bis zum Ende derselben schwindet, um am Tage post partum wiederzukehren und 1 Jahr lang zu bestehen. 2. Fall. Auftreten kurz vor Schlub der 1. Gravidität, bessert sich post partum und heilt ab. 2. Gravidität normal, 3. Gravidität neuer Ausbruch im 3. bis 4. Monat, der bis zum Ende der Gravidität schwindet, aber am 3. Tage post partum wiederkehrt und 2 Monate dauert. Ebenso in 2 anderen Fällen heftige Nachschübe am 1. und 5., resp. am 4. Tage post partum.

Bataille (25): Ausbruch am Tage nach der 5. Eatbindung nach normaler Schwangerschaft; in der folgenden Schwangerschaft Beginu des Ausschlags im 4. Monat, äbnlich in einem Fall von Perin (26), von Canuet (27), wo der Ausschlag zuerst zwei Tage nach der 5. Entbindung auftrat und 4 Wochen dauerte, in der 6. Schwangerschaft im 4. Nonat begann, mit Nachschüben bis zur Entbindung dauerte und sich danach verschlimmerte und 5 Wochen post partum aushielt; in der 7. Schwangerschaft vom 6. Monat bis zur Entbindung, in der 8 . schon vom 2. Nonat an mit sehr heftigem Nachschub 48 Stunden post partum.

Die Erklärung dieses Aufflammens des Krankheitsprozesses im Wochenbett ist durch die obigen Befunde, welche für das schubweise Eintreten von Fermenten bei den Involutionsvorgängen nach der Entbindung sprechen, gegeben. Bietet doch überhaupt der ganze Prozeß der Imp. herp., aber auch der anderen Schwangerschaftsdermatosen große Ähnlichkeiten mit dem Bilde der Fermentintoxikation.

Es ist ohne weiteres einleuchtend, $\mathrm{da} \beta$ die Erscheinungen auf der Haut nicht als das̀ beherrschende Moment der ganzen 
Krankheit, sondern nur als ein Symptom einer Intoxikation aufgefaßt werden müssen, welche sich durch die verschiedensten Erscheinungen kund gibt. Als solche sind zu betrachten:

1. Das plötzlich mit Schüttelfrösten einsetzende Fieber, welches ebenso steil wieder zur Norm abfällt, um sich in gleichen Schüben zu wiederholen.

2. Die Erscheinungen vom Magendarmkanal: Erbrechen, Diarrhoen, auch blutige.

3. Die Erscheinungen ron seiten des Zentralnervensystems; außer dem oft enormen Jucken und Brennen findet sich fast stets bei den Kranken eine große psychische Erregung welche z. B. in unserem Fall schon vor Auftreten des Exanthems auffällig war - welche sich selbst bis zu Delirien steigern kann.

Ebenso sind Schwindel, Ohnmachten, Koma, eklamptische Anfälle (Breier), Kontrakturen, Nystagmus etc. beobachtet.

4. Die hochgradige Schwäche, die auch in den zunächst günstig verlaufenden Fällen eintretende starke Abmagerung und der meist nach wochenlangem Bestehen der Krankheit eintretende Tod.

Sehr analog tritt im Tierversuch - auch bei Einführung steriler Fermente - Fieber, Zittern, Unruhe, taumelnder Gang, Abmagerung, Schwäche, endlich auch Koma auf; ein Teil der Tiere erholt sich, während ein anderer Teil erst nach Wochen stirbt (Kionka) (28). Auch beim Menschen hat Hildebrandt (29) durch subkutane Injektion von Labferment und durch Einverleibung von Myrosin per clysma steil ansteigendes Fieber erzengt.

Der in unserem Fall erhobene Befund von Amyloid spricht gleichfalls in gewissem Sinue für eine Fermentintoxikation, da wir durch die Experimente Schepilewskys (30) wissen, daß besonders Labferment, aber auch Pankreatin und Papayolin bei Kaninchen ohne Mitwirkung von lebenden Bakterien oder von Bakterienprodukten Amyloid erzeugt.

Leider war zu der Zeit, wo unser Fall das erstemal in unserer Beobachtung war, der erhöhte Antitrypsingehalt in der Schwangerschaft noch nicht bekannt; es erscheint daher nötig, daß andere Beobachter in künftigen Fällen ihre Aufmerk- 
samkeit darauf richten, ob etwa bei der Impetigo herpetiformis dabei besondere Verhältnisse vorliegen. Wir haben versucht, ob das Serum der Patientin mit einem Plazentarextrakt (Georg Michaelis) eine Komplementablenkung ergäbe, aber keinen positiven Ausschlag gefunden; doch miißten in Zukunft auch nach dieser Richtung weitere Versuche gemacht werden. ${ }^{1}$ ) Man muß sich jedoch vorstellen, daß nicht der bloße Eintritt von Fermenten, auch nicht eine übergroße Menge dieser, das Krankheitsbild auslöst, sondern daß die Organe, deren Aufgabe es ist, die von der Plazenta aufgenommenen Toxine unschädlich zu machen und an welche in der Schwangerschaft sehr hohe Anforderungen gestellt werden, insuffizient werden.

So scheint ja während der Gravidität überhaupt die antitoxische Funktion der Leber herabgesetzt zu sein (siehe das Nähere bei $\mathrm{H}$ of bauer) (31); ebenso fällt den Nieren in dem Entgiftungsprozesse eine große Bedeutung zu; ihre Aufgabe besteht in der Ausscheidung der Toxine, insofern sie den Destruktions- und Bindungsvorgängen in der Leber nicht anheimgefallen sind (van Leersum) (32). Es droht die Gefahr, $\mathrm{daB}$ die Überanstrengung dieser Organe zu einer Insuffizienz, sogar zu einer Beschädigung des Parenchyms führen kann, welche auch in histologischen Veränderungen kenntlich sein kann. In diesem Sinne kann die bei Imp. herp. sehr häufig konstatierte trübe Schwellung der Leber und Niere zwanglos gedeutet werden, welche ihre Analogie hat in den Befunden nach experimenteller Injektion von Plazentafermenten bei Kaninchen (Hofbauer, p. 247, Liepman n-Weichard t l.c). Bei dieser Anomalie erleidet ja überhaupt die funktionelle und assimilatorische Funktion der Zelle EinbuBe und es geht auch eine gesteigerte Einschmelzung des Plasmaleibes vor sich, welche $\mathrm{Hofbauer}$, gestiitzt auf die Arbeiten von J acoby,

1) Der negative Ausfall ist allerdings kein Beweis gegen Veränderungen des Fermenttiters im obigen Sinn, da man Komplementablenkung bei dem System Ferment und Antiferment bisher nur bei Verwendung artfremder Fermente, bei welchen also die Ablenkungsreaktion schon durch das artfremde Eiweiß und seine Antikörper bedingt sein kann, gefunden hat. 
Magnus Levy, Levene, als eine fermentative Autolyse auffaßt. Diese autochthonen Fermente kommen aber, da das Serum normalerweise eine hemmende Wirkung auf intrazelluläre Fermente entfaltet, nach Jacoby besonders dann in erhöhtem Maße zur Geltung, wenn ein Plus von Fermenten aus anderen Organen ihnen zugeführt wird.

An ähnliche Vorgänge kann man bei den Schwangerschaftsdermatosen denken; an eine Insuffizienz der Leber und Niere gegenüber den in den maternen Kreislauf eintretenden - vielleicht auch anormal hochdosierten - Plazentarfermenten. In diesem Sinne spricht auch der Umstand, daß eine grobe Zahl von den an Imp. herp. Erkrankten auffällig viele Schwangerschaften in wenigen Jahren durchgemacht haben, z. B. die Fälle von Borzecki 7 Graviditäten, Schultze 9 Graviditäten, Groß 7 Graviditäten, $\mathrm{Kren} 7$ Graviditäten und viele andere, und z. T. auch Funktionsstörungen der Leber darboten.

Erwähnenswert ist auch, daß wir in unserem Fall kurz vor dem Tode im Urin und dann noch in der Leber und den Nieren Quecksilber fanden (Prof. Loeb), während die Patientin solches bestimmt in den letzten Monaten weder bei uns noch bei Kollegen Rosenthal erhalten hatte; es scheint dies für eine Insuffizienz der ausscheidenden Organe zu sprechen.

Sehr in Betracht zu ziehen ist aber fernerhin, besonders in Rücksicht auf die manuigfachen zerebralen Symptome bei den Schwangerschaftsdermatosen, ob die von Erdheim und Stumme (33) geschilderten Schwangerschaftsveränderungen der Hypophyse, welcher gleichfalls eine entgiftende Funktion speziell in der Schwangersehaft zukommt, der Norm entsprechen.

Auch das vielfach bei Imp. herp., Herpes gestationis beobachtete Einsetzen der Rezidive in stets früheren Zeitpunkten der Schwangerschaft, sowie die damit verknüpfte auffällige Steigerung der Heftigkeit der Symptome spricht für eine zunehmende Insuffizienz der entgiftenden Organe. Außerdem ist dabei jedoch bei derartigen, zu verschiedenenmalen schubweise eintretenden Fermentintoxikationen an Anaphylaxie zu denken, wie sie auch bei experimentellen Fermenteinverleibungen zu Tage tritt und diese Arbeiten wesentlich erschwert. 
Wir kommen zu der Annahme, daß ähnliche Schädigungen durch die geschilderten nicht genügend entgifteten Toxine, wie die Nieren auch die Haut, welche ja als Ausscheidungsorgan, besonders bei Schädigung der Nieren, funktioniert, erleidet und dadurch nekrotisierende Vorgänge, besonders im Epithel, zu Stande kommen, welche ihren klinischen Ausdruck in den sterilen Pustelchen finden. Dafür spricht klar, daß der geringe Druck des Gummischlauches die Pusteln früher auftreten ließ, d. h. daß schon ein mäßiger Reiz in der geschädigten Haut nekrobiotische Prozesse herbeiführte. Wenn wir uns auch klar darüber sind, daß die vorgetragene Ansicht im wesentlichen einen hypothetisch-theoretischen Gedankengang zur Grundlage hat, so hielten wir doch bei der absoluten Dunkelheit des Gebietes die Aussprache eines solchen für gerechtfertigt, weil er für die Erforschung der Ätiologie späterer Fälle wenigstens eine gewisse Fragestellung und Untersuchung ermöglicht. In späteren Fällen müßte, abgesehen von der ja schon sicher erscheinenden Frage nach der Sterilität des Pustelinhaltes und Blutes, geklärt werden:

1. die Frage nach dem Antitrypsingehalt des Serums nach Ausbruch des Exanthems und vor allem nach Eintritt der Geburt, sowie auch in- und außerhalb der Fieberattacke;

2. müßten Komplementbindungsversuche mit Plazentarpulver resp. Enzymen fortgesetzt werden, eventuell auch die kutane Empfindlichkeit gegen Plazentarfermente geprüft werden;

3. müßte auf Zottendeportationen und Deziduommetastasen,

4. auf Veränderungen der Hypophysis und Thymus genau geachtet werden.

Vielleicht gelingt es dann, langsam etwas mehr Licht auf die Pathologie dieser dunklen Krankheiten za werfen. 


\section{Literatur.}

(Siehe bei Scherber und Gavazzeni.)

1. Scherber. Arch. f. Derm. u. Syph. XCIV. Bd. p. 227.

2. Hebra. Wiener med. Woch. 1872. Nr. 48.

3. Kaposi. Arch. f. Derm. v. Syph. XIX. Bd. p. 273.

4. J ar is c h. Hautkrankheiten. 1900. p. 408.

5. Gavazzeni. Monatsh. f. pr. Derm. Bd. XLIX. p. 8.

6. Vidal. Considerations sur la dermatose gravidique autotoxique. Thèse Paris 1906.

7. Canuet. Ann. de derm. et syph. 1896. p. 384.

8. Perrin, Charles. De la dermatite de Duhring au cours de la grossesse. Thèse Paris 1895.

9. Schmorl. Pathologisch-anatomische Untersuchungen über die puerp. Eklampsie. Leipzig 1893.

10. Veit. Verschleppung der Chorionzotten.

11. Zentralbl. f. Gynäjol. 1902, $\mathrm{N}^{\mathrm{x}}, 7$.

12. Münch, med. Woch, 1902. Nr. 44 und Hygienische Runḋschau. 1903. Nr. 10.

13. Münch. med, Woch. 1905. Nr. 51.

14. Hofbauer. Grundzüge einer Biologie der menschl, Plazenta. Wien und Leipzig. 1905.

15. Gräfenberg. Beiträge zur Physiologie der Eieinbettung. Zeit. schrift f. Geburtsh. u. Gynäkol. Bd. XLV.

16. Zur Kenntnis der Plazentaeekzeme. Biuchemische Zeitschrift. Bd. XXII. H. 3/4. p. 316 ff.

17. Ebenda. p. 337.

18. Münch. med. Woch. 1909. Nr. 22.

19. Über die Bedeutung des proteolytischen Leukozytenfermentes im Lochialsekret und im Kolostrum, sowie über den Antitrypingehalt im Serum der Wöchwerinnen. Archiv f. Gynälologie. Bd. LXXXIX. H. 3.

20. Arch. f. Derm. u. Syph. 1873. V. Jahrg. 169.

21. Annales de derm. et syph. 1892.

22. Brooq. Über die Dermatitis herpetiformis Duhrings. IV. Teil. Monatsh. f. pr. Derm. IX. Bd. p. 20.

23. Köbner. Über das Vorkommen von Pemphigus acutus. Archiv f. Derm. u. Syph. Bd. I. p. 209.

24. Lesser. Dermat. Zeitschr. Bd. VI. p. 206. Buschke. Charité Annalen. 24. Jahrgang. 
25. Annales de derm. et syph. 1892. p. 648 .

26. Ebenda. 1894. p. 1348.

27. Ebenda. 1896. p. 384.

28. Deutsche med. Woch. 1896. p. 612.

29. Virchows Archiv. Bd. CXXI. H. 1 und Bd. CXXXI. p. 5.

30. Schepilewsky. Exper. Beitr. z. Frage der amyloiden Degeneration. Zentralbl. f. Bakt. 1899. XXV. Nr. 24. p. 849.

31. Ho fba uer. Beiträge zur Ätiologie und Klinik der Graviditätstoxikosen. Zeitschr. f. Geburtshilfe u. Gynäkol. Bd. LXI.

32. van Leersum. Ausscheidung von Aminosäure während der Schwangersehaft und nach der Geburt. Biochem. Zeilsehr. Bd. XI.

33. Erdheim und Stumme. Schwangerschaftsveränderungen der Hypophyse. Zieglers Beiträge. Bd. XLVI. p. 1 ff. 
Archiv f. Dermatologie U. Syphilis Band CII.
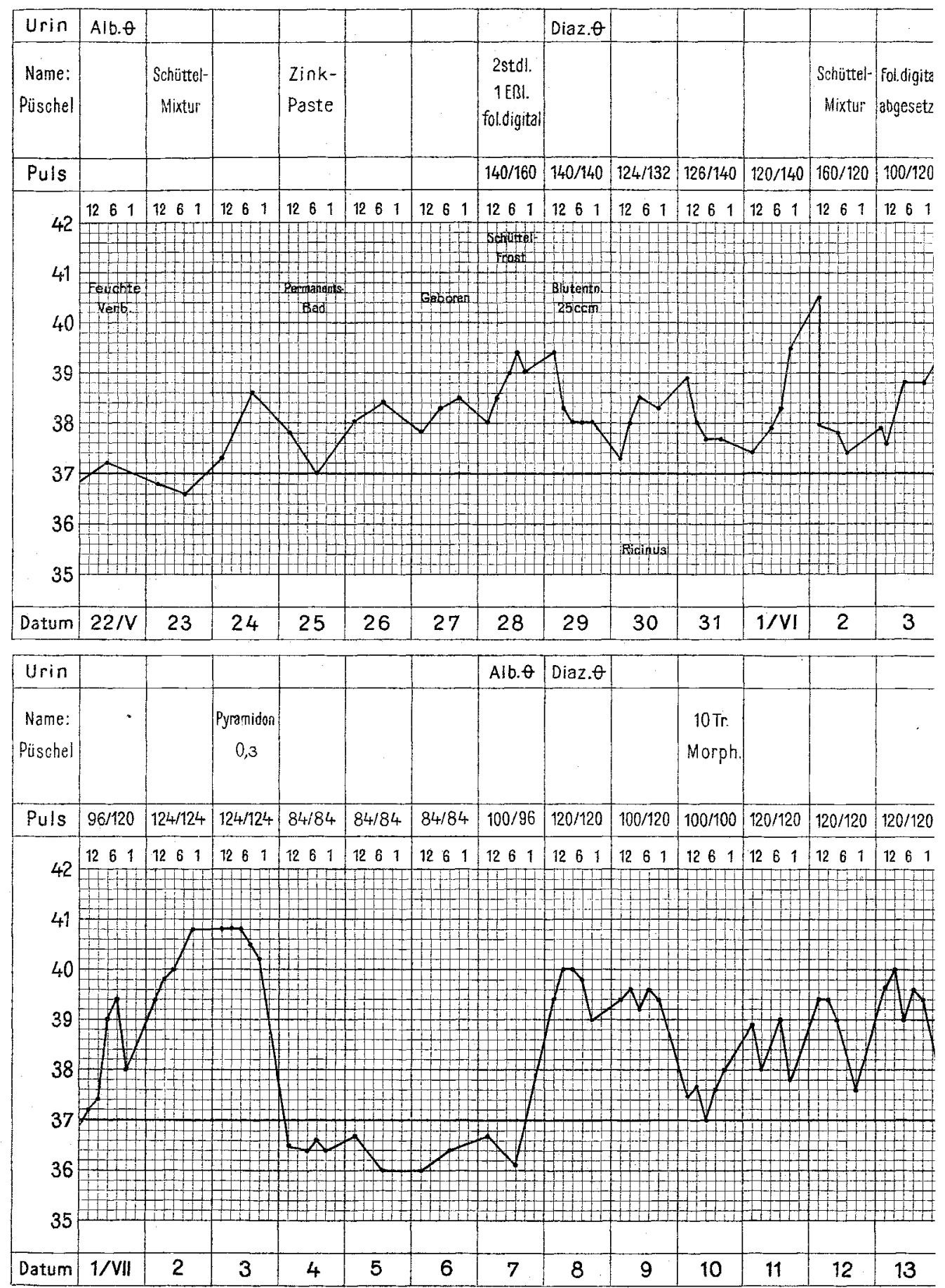

Wechselmann: Impetigo herpetiformis gravidarum Hebra. 